\title{
Analysis of Local Macroeconomic Early-Warning Model Based on Competitive Neural Network
}

\author{
Xiaoxuan Wang, ${ }^{1}$ Jingjing Wang $\mathbb{D}^{2},{ }^{2}$ Ying Zhang, ${ }^{3}$ and Yixing $\mathrm{Du}^{4}$ \\ ${ }^{1}$ National School of Development, Peking University, Beijing 100089, China \\ ${ }^{2}$ Hebei College of Science and Technology, Baoding 071000, China \\ ${ }^{3}$ Operation Office, The People's Bank of China, Beijing 100089, China \\ ${ }^{4}$ Investment Banking Department, China International Capital Corporation Limited, Beijing 100089, China \\ Correspondence should be addressed to Jingjing Wang; wangjingjing@heyangedu.com
}

Received 20 December 2021; Revised 15 January 2022; Accepted 17 January 2022; Published 11 February 2022

Academic Editor: Miaochao Chen

Copyright $\odot 2022$ Xiaoxuan Wang et al. This is an open access article distributed under the Creative Commons Attribution License, which permits unrestricted use, distribution, and reproduction in any medium, provided the original work is properly cited.

\begin{abstract}
At present, the commonly used index selection methods for macroeconomic early-warning research include K-L information volume, time difference correlation analysis, and horse farm methods. These traditional statistical methods cannot cope with the continuous changes of economic indicators, and due to the existence of statistical errors, these methods are difficult to perform. Therefore, this paper proposes to use a self-organizing competitive neural network to select early warning indicators. Its selflearning and adaptive characteristics and fault tolerance overcome the limitations of the above statistical methods. This article proposes a method of selecting macroeconomic early-warning indicators using self-organizing competitive neural networks and designs a macroeconomic nonlinear early warning model of self-organizing competitive neural networks; using fuzzy logic reasoning to introduce economic experts' experience into macroeconomic early warning analysis, the system has the ability to deal with nonlinear and uncertain problems and realizes the intelligence of the early-warning process, uses the national macroeconomic indicator data from January 1997 to March 2008 for empirical analysis, and compares the self-organizing competitive neural network method with the traditional KL information method. From the experimental results, compared with the KL information method, the self-organizing competitive neural network method selects more comprehensive indicators and has greater advantages in seismic resistance and stability.
\end{abstract}

\section{Introduction}

Macroeconomic early warning is based on realistic or predicted macroeconomic indicators to make judgments on current and future economic operations. The traditional early warning signal system design is to select a set of sensitive indicators that reflect the economic development status and merge them into comprehensive indicators; red, yellow, and green lights are used similar to a set of traffic control signals to control this set of comprehensive indicators. The reflected current and future economic conditions give different signs and signals. By observing the changes in the signals, we can judge the current movement status and future development trends of the economic system.
However, due to the nonlinearity and uncertainty of the macroeconomic system, the linear early-warning model in the traditional sense has a large error with the actual system. How to study the nonlinear problems in the macroeconomic system and how to integrate the experience accumulated by economic experts for many years into the study of future economic trends is an understanding problem that needs to be solved in economic early warning and even macroeconomic research, and it is an understanding of complex systems. Correctly understanding the problem in economic development, stable development, and suppression of fluctuations is a major goal of macroeconomic policies. However, the expansion of the reproduction cycle involves all aspects and links of economic activities. Once a certain 
aspect or link has unconventional changes, it may lead to overall economic fluctuations. Moreover, the factors that cause and induce changes in individual aspects and links are ever changing, so it is difficult for people to fundamentally eliminate economic fluctuations. However, people can take effective measures to slow down macroeconomic fluctuations, extend the period of economic growth, and shorten the period of economic recession by understanding the status of macroeconomic operations and predicting future macroeconomic operations so as to promote healthy and stable economic growth. Therefore, research on monitoring and early warning of economic fluctuations has been an issue of great concern to scholars in economic circles at home and abroad [1-10]. It is very necessary to properly establish an economic monitoring and early warning mechanism and to regulate and control abnormal conditions in economic operations in a timely manner. This paper uses neural network theory, fuzzy system theory, and other nonlinear analysis tools to study the macroeconomic earlywarning system from a brand-new perspective and puts forward the "fuzzy intelligence" early-warning idea, which combines the model theory with the neural network to establish the macroeconomic nonlinear early warning system. Fuzzy logic reasoning is used to introduce expert knowledge into the macroeconomic early warning process so that the system has the ability to deal with nonlinear and uncertain problems and realizes the intelligence of early warning; the self-learning, self-adaptation, and generalization capabilities of the neural network are used to complete the realization and operation of the system. The neural network is shown in Figure 1.

Artificial intelligence is considered to be a "discipline about knowledge" due to its comprehensiveness of disciplines, and due to its integration of high-precision technology, it is considered to be used to realize intelligent activities that replace humans with machines, which also represents the relevant center and core idea of the field. That is, through the frame-by-frame study of human motion trajectories, many commands are given to the machine, and even the machine's own knowledge system is cultivated to replace humans in handling some behaviors/businesses. The research content is mainly to control the machine through code to imitate and learn the characteristics of humans. The act of consciousness conducts learning and knowledge theory and operation.

As the core of artificial intelligence disciplines, machine learning is the basis for making computers intelligent. It has interoperability and tolerance of disciplines and can organically integrate multiple types of theories. The main content is to use algorithms to enable the machine to have a certain simulation ability, to independently complete some behaviors that only humans can complete before, to establish its own knowledge structure and skill system, and to update and improve its knowledge structure in real time. As one of the most important technologies of artificial intelligence, it is the basis for the realization of mechanical intelligence. Generally speaking, machine learning is mainly used to summarize and integrate information, which is then deduced by artificial intelligence [11-15].
In recent years, the artificial neural network has received widespread attention. It is established to simulate the structure and thinking form of the human brain. The human brain is composed of tens and thousands of complex neurons, which are connected by a synaptic system. Therefore, compared with computers, the human brain has powerful analytical and judgment capabilities, associative memory capabilities, parallel processing capabilities, and recognition capabilities. Because the human brain has many superior performances, people want to build a similar artificial neural network to solve some complex problems by simulating the working principle of the human brain. The research of the neural network has received extensive attention from people from all walks of life. Researchers in different fields want to use the function of neural network to solve the problems they are facing. In the future, artificial neural network theory will have more room for development and greatly promote science and technology [16-20].

In recent years, neural networks have developed rapidly. Geoffrey Hinton used a layer-by-layer training method to build a deep learning network. This technology can greatly improve the effect of the generalization model on the test set and has attracted the attention of many people. In 2009-2012, the artificial intelligence research team led by Jürgen Schmidhuber developed recurrent neural networks and deep feedforward neural networks. At the ImageNet conference in 2012, Geoffrey Hinton research members applied deep learning-related technologies to image classification, which made it far more effective than the second, which inspired scholars' enthusiasm for deep learning research in recent years. China has also organized many largescale international conferences on neural networks. Neural network research has received widespread attention from people from all walks of life. Researchers in different fields want to use the functions of neural networks to solve their problems. The problems facing artificial neural network theory will have more room for development in the future, which will greatly promote science and technology.

With the continuous development of neural networks, many difficulties have also caused many scholars to question it. In this stagnant period, the perceptron network proposed by Minksy and Sparpcrt broke the deadlock at the time and produced profound effects on the neural network influence. However, during the low period of research at that time, many scholars have been continuously studying it. Sgrossberg and GA Carpenter proposed the ART model in 1969. In 1972, the Finnish scholar Tkohonen researched and proposed an unsupervised learning neural network. It is called the self-organizing mapping theory network (SOM model). In 1982, American physicist Hopfield proposed a new neural network model, which was later called the Hopfield neural network model [7]. In this model, it redefines that the "energy function" also gives the criterion of network stability, which makes the Hopfield network have the ability of associative memory and optimization solutions, which opens a new door for subsequent research in this area and also makes the research on neural networks renewed. After gaining popularity, Bell Labs used the working principle of the Hopfield neural network to design the first neuron 


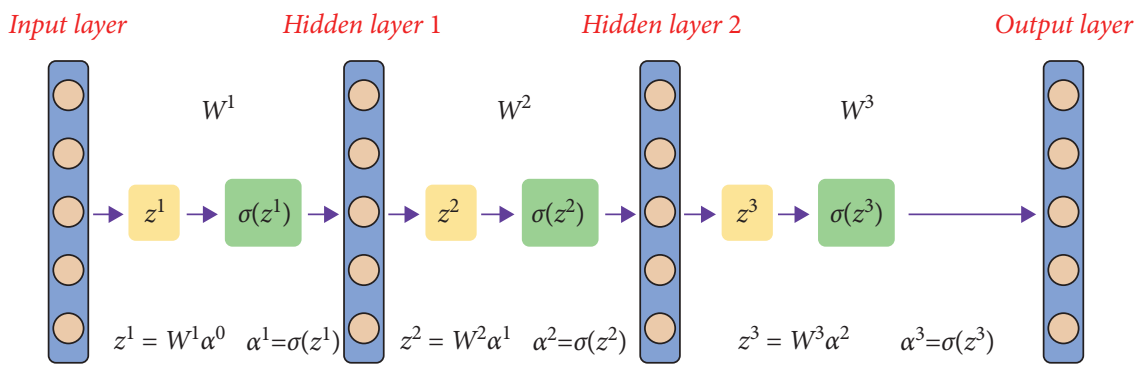

Figure 1: Neural network.

network with silicon chip as hardware. GEHinton and TJSeinowski used the principle of simulated annealing to make the energy of the entire network reach the global minimum. This can overcome the problem and limitations of the Hopfield network. We call this kind of network a "Boltzmann machine." In 1987, scholars from all over the world were invited to participate in the first neural network conference held, and the first neural network association was also established [20-25].

As we all know, Meyer-Brue proposed an unsupervised learning network, which is called a "competitive neural network." There are two types of state variables in this model. The first type is short-term memory (STM) state variables that describe rapid neural activity, and the other type is long-term memory (STM) state variables that describe slow unsupervised synaptic modification. Both the competitive neural network and the Hopfield network model can automatically classify input patterns through learning, but there are also some different aspects. The competitive neural network is composed of the input layer and the competition layer, and the neurons are both in the input layer and the competition layer, that is, the two-way connection between the two layers; at the same time, the neurons can also be horizontally connected between the same layer. The working mechanism of the competitive neural network is to let the competition layer compete for the input signal, and finally, only one winning neuron will output the signal. There are two ways of competition in the competition layer: one is ordinary competition; that is, every neuron in the competition layer has competition with other neurons; the other is called lateral inhibition; that is, only the neurons in contact with oneself are inhibited, and the method of lateral inhibition competition can solve the problem of clear image edges [26-29].

The economic early-warning system is an indicator or demander that can reflect the trend and magnitude of economic development in advance of the macroeconomic operation process. It can send output signals in advance when the economy is overheating, downturn, and recession. A system formed by a set of index systems is established based on macrocontrol. In recent years, many scholars have conducted research on it. Duan et al. have established new time-delay-independent criteria by using inequality techniques to ensure the finite-time synchronization of the considered competitive neural network. Luo and Cui designed a class of state feedback controllers to study the synchronization problem of competitive neural networks. Liu et al. proposed a fractional-order competitive neural network with multiple time-varying delays and discussed the global asymptotic stability of this type of neural network. A new generalized inequality is given, and the theoretical results provide a new perspective for proving the close relationship between the network stability criterion and the network topology. $\mathrm{Gu}$ used an adaptive control method to study the complete synchronization of coupled time-delay competitive neural networks with different time scales and random disturbances. Yang et al. studied the adaptive lag synchronization problem of competitive neural networks with mixed time delays and mixed disturbances by designing robust adaptive controllers. Gan et al. used an adaptive method to consider the synchronization problem of random interference and delay competition neural networks with unknown parameters. $\mathrm{Gu}$ et al. used the nonlinear Lipschitz measure (NLM) method to construct a suitable Lyapunov function and obtained several sufficient conditions for the existence of the unique equilibrium point and the global exponential stability of the scale and multidelay competitive neural network. Meyer-BaseA proved the local uniform stability of a class of multi-timescale neural networks with uncertain parameters and determined that the stability condition is a simple relationship between neural parameters. It is assumed that the obtained parameter disturbance is only limited by its boundary. Meyer-BaseA et al. proved that in most computational models of competitive neural networks, activities at different time scales interact. The existing model is extended by considering the stochastic process, and the stability result is established on the basis of stochastic system theory. Nie and Cao use the two general activation functions to study the multi-stability of competitive neural networks with time-varying and distributed delays. By constructing parameter conditions and using inequality techniques, it is derived that there is a $3 \mathrm{~W}$ equilibrium point, where the equilibrium point is a necessary and sufficient condition for exponential stability. Meyer-BaseA established the robust stability results of competing neural networks with different time scales under parameter perturbation and certain conditions, 
assuming that the uncertainty of the system is limited by the upper bound of its norm.

\section{Macroeconomic Early Warning Model Based on Fuzzy Neural Network}

Competitive neural networks have attracted much attention from scholars because of their unique competitive mechanism and good performance in image recognition and data processing. Therefore, it is of great significance to analyze the dynamics of competitive neural networks. When designing an early warning signal system, we must first select a set of sensitive indicators that reflect economic development and then use relevant data processing methods to combine multiple indicators into a comprehensive indicator and pass a set of traffic control. The red, yellow, and green signal signs send different signals to the current economic conditions of this group of indicators and comprehensive indicators. Finally, by observing and analyzing the changes in the signals, we can judge the trend of future economic growth.

2.1. Selection of Early Warning Indicators. The most important task of establishing an early warning signal system is to select macroeconomic early warning indicators. Early warning indicators should be able to reflect the overall scale, level, and speed of development of the national economy in different aspects. The indicators of candidates should meet the following conditions: (a) the selected indicators must be economically important, and the selected indicators must collectively represent the main aspects of economic activities; (b) consistency or advancement, that is, generally consistent with or slightly ahead of economic cycle changes, can be sensitive to reflecting the dynamics of the scene; (c) statistical rapidity and accuracy.

Based on the above principles and combined with my country's actual conditions, this article selects the following eight economic early warning indicators:

Industrial value added, total energy production, cash in circulation $M$, narrow money supply $M$, total retail sales of consumer goods, balances of various deposits of financial institutions, balances of various loans of financial institutions, and retail price indices of commodities. The network structure diagram is shown in Figure 2.

2.2. Composition of Early Warning Signals. The early warning system signal is composed of a set of alert indicators assigned with different colors, usually indicated by signal lights, and there are five types: "red light," "yellow light," "green light," "light blue light," and "blue light." When the TC sequence of the growth rate of the indicator exceeds a certain check value, the corresponding signals are displayed, respectively. If the signal lights up with a "green light," it means that the economic development at that time is very stable, and the government can take control measures to promote economic growth while stabilizing. The "red light" means that the economic development is "overheated." At this time, fiscal and financial institutions should adopt austerity measures to gradually restore the economy to normal conditions. The "yellow light" indicates

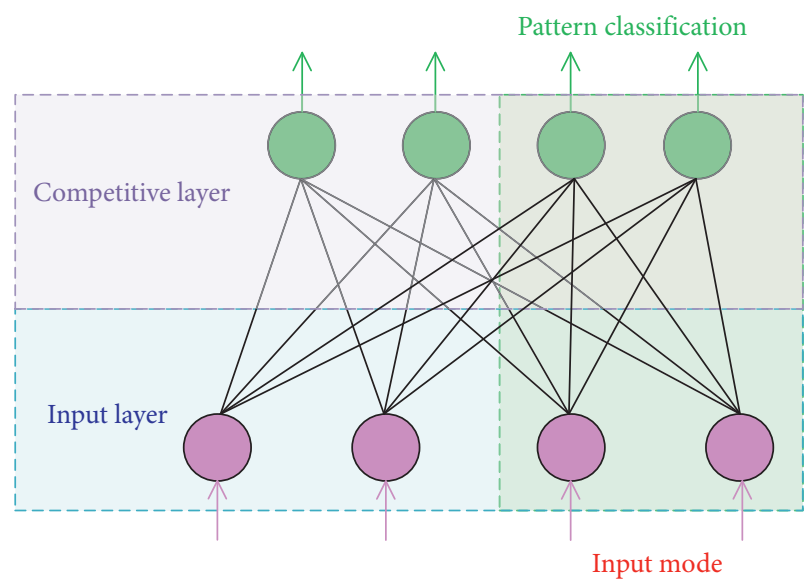

FIgURE 2: Network structure diagram.

that the economy is still stable and economic growth is "slightly hot," and there is a possibility of a turnaround and stabilization in the short term. The "light blue light" indicates that the economy may stabilize or shrink in the short term. The "blue light" indicates that the economy is in a state of contraction or depression.

At the same time, 5 kinds of signals are scored, such as "red light" 5 points, "yellow light" 4 points, "green light" 3 points, "light blue light" 2 points, and "blue light" 1 point. If $N$ early warning indicators are selected and each indicator is given a corresponding score in each period, the score of the comprehensive indicator can be obtained. When all indicators are red lights, the combined score is the highest $5 x$ points. When all lights are blue, the overall score is the lowest $N$ score. Then, through the check value of the comprehensive score, it is comprehensively judged which kind of light should be on for the warning signal of the current month.

2.3. Determination of Early Warning Limits. Each selected index sequence is compared with the value of the same month of the previous year to obtain the growth rate sequence; then, the growth rate sequence is seasonally adjusted, and the adjusted TC sequence is taken to formulate the early warning signal. The early warning limit is also called the check value, which refers to the boundary between two adjacent signal lights of 5 types of signal lights. Whether the determination of the early warning limit is appropriate is of vital importance and is related to the correct judgment of the operation of the entire national economy. Therefore, it must be determined carefully. Usually, the determination of the early warning limit adopts the method of empirical judgment. The value variation is shown in Figure 3.

Since the industrial value-added indicator is basically the same as the economic cycle changes, the early warning limit of the industrial value-added indicators is determined first. the average growth rate of industrial added value is taken as $169 \%$ in the three months before the peaks of the two economic cycles since 1987 as the boundary between the red-light district and the yellow-light district. the average growth rate of industrial added value is taken as $37 \%$ in the first three months of the bottom of the two economic cycles 
since 1987 as the boundary between the light blue light area and the blue light area.

Generally speaking, economic indicators are time-series indicators, so using the BP neural network to predict them can be regarded as forecasting time series. Research in this area has a very deep theoretical foundation. Suppose a univariate time series is described as follows:

$$
X_{i+k}=f\left(X_{1}, X_{t-1}, \ldots, X_{t-m+1}\right),
$$

where $X$ is a univariate time series.

A neural network is used to fit this functional relationship, and it is used to derive future values. This is the basic idea of using artificial neural networks for time-series forecasting. The data comparison is shown in Figure 4.

The purpose of selecting the average value of the growth value of the previous 3 months is to be able to report to the police in time before the economic growth reaches overheating or shrinking. The three-month interval is determined based on the characteristics of my country's short economic cycle but large volatility. When the BP neural network is used to predict the economic time series, the input and output data must first be preprocessed and turned into data between $[-1,1]$. The preprocessing of input and output data is a key step for effective training of neural networks, which directly affects the performance of the trained network. The method used in this article is to normalize the original data, that is, to uniformly limit the input and output data within the interval $[-1,1]$ through a certain linear transformation. The data processing is as follows:

$$
\widehat{x}=\frac{x-x_{\min }}{x_{\max }-x_{\min }}
$$

where $X$ is the processed value, $x$ is the value of the original sequence, $x_{\min }$ is the minimum value in the sequence, and $x_{\max }$ is the maximum value in the sequence.

2.3.1. Network Model Design. The basic principle of using the self-organizing competitive neural network to select indicators is to normalize the indicators to be selected as the input of the self-organizing competitive neural network; for the same input mode, the output value of the network is also the same so that the indicators are correctly divided into the leading indicator group, the consistent indicator group, and the lagging indicator group. The self-organizing competitive neural network is composed of an input layer and a competition layer. The input layer has $N$ neurons, and the competition layer has $M$ neurons. The connection rights between the input layer and the competition layer are as follows:

$$
\left\{\left|W_{i j}\right|\right\}, i=1,2, \ldots, N ; j=1,2, \ldots, M \text {. }
$$

The corresponding constraints are as follows:

$$
\sum_{i=1}^{N} W_{i j}=1
$$

In the competition layer, neurons compete with each other, and finally, only one or a few neurons win to adapt to the current input sample. The neuron that wins the competition represents the classification mode of the current input sample. The Tinput learning modes of the network are as follows:

$$
P_{k}=\left(p_{1}^{k}, p_{2}^{k}, \ldots, p_{N}^{k}\right) \text {. }
$$

The corresponding output mode of the competition layer is

$$
A_{k}=\left(a_{1}^{k}, a_{2}^{k}, \ldots, a_{N}^{k}\right), \quad k=1,2, \ldots, T .
$$

2.3.2. Learning Algorithm. The learning steps of self-organizing competitive neural network are as follows:

(1) Initialization. According to the constraint condition of formula (4), the random value in the following formula is assigned. The prediction is shown in Figure 5.

$$
\left\{W_{i j}\right\} \in[0,1], \quad i=1,2, \ldots, N ; j=1,2, \ldots, M
$$

(2) Choose one mode $P^{k}$ among $T$ learning modes and provide it to the input layer of the network.

(3) Calculate the input value of each neuron in the competition layer according to the following formula:

$$
S_{j}=\sum_{i=1}^{N} W_{i j} p_{i}^{k}, \quad j=1,2, \ldots, M .
$$

(4) According to the principle of "the winner is king," the neuron corresponding to the maximum value in $S_{j}(j=1,2, \ldots, M)$ is taken as the winner, and its output state is set to 1 , and all other neurons' output state of the element is set to 0 , namely,

$$
\begin{aligned}
& a_{j}=1, S_{j}>S_{i}(i \neq j), \\
& a_{i}=0, i \neq j .
\end{aligned}
$$

If the phenomenon of $S_{j}=S_{i}$ occurs, the neuron on the left is selected as the winning neuron according to the unified agreement.

(5) Each connection weight connected to the winning neuron is modified according to the following formula, while all other connection weights remain unchanged.

$$
w_{i j}=w_{i j}+\Delta w_{i j}
$$

$\Delta w_{i j}=\eta\left(\frac{P_{i}^{k}}{m}-w_{i j}\right), \quad i=1,2, \ldots, N(0<\eta<1)$.

Among them, $\eta$ is the learning rate, and $m$ is the number of elements 1 in the $k$-th learning mode.

(6) Choose another learning mode and return to step (5) until all $T$ learning modes are provided to the network. 

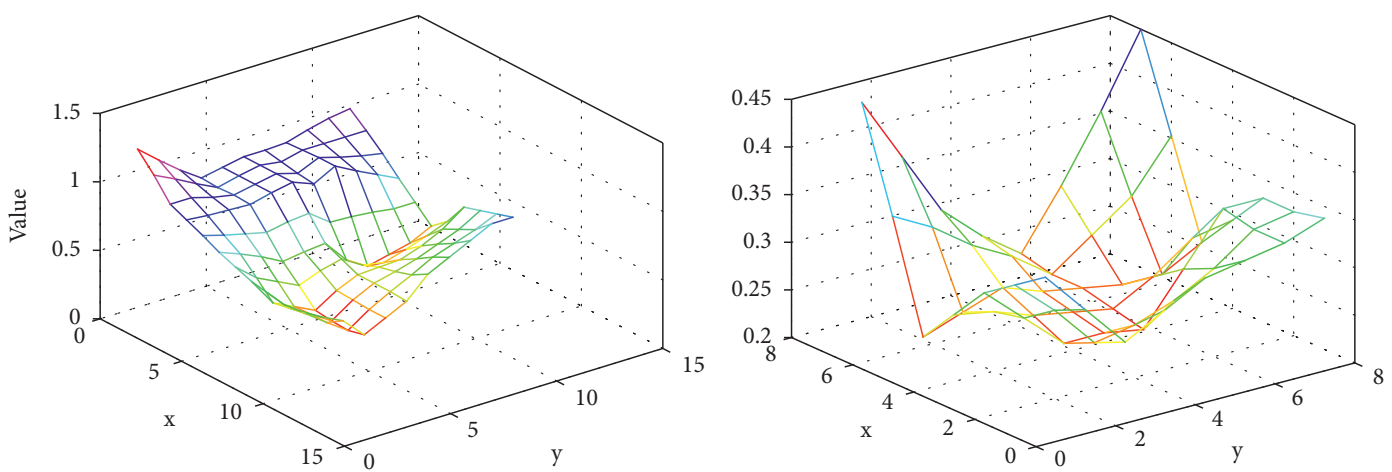

Figure 3: Value variation.
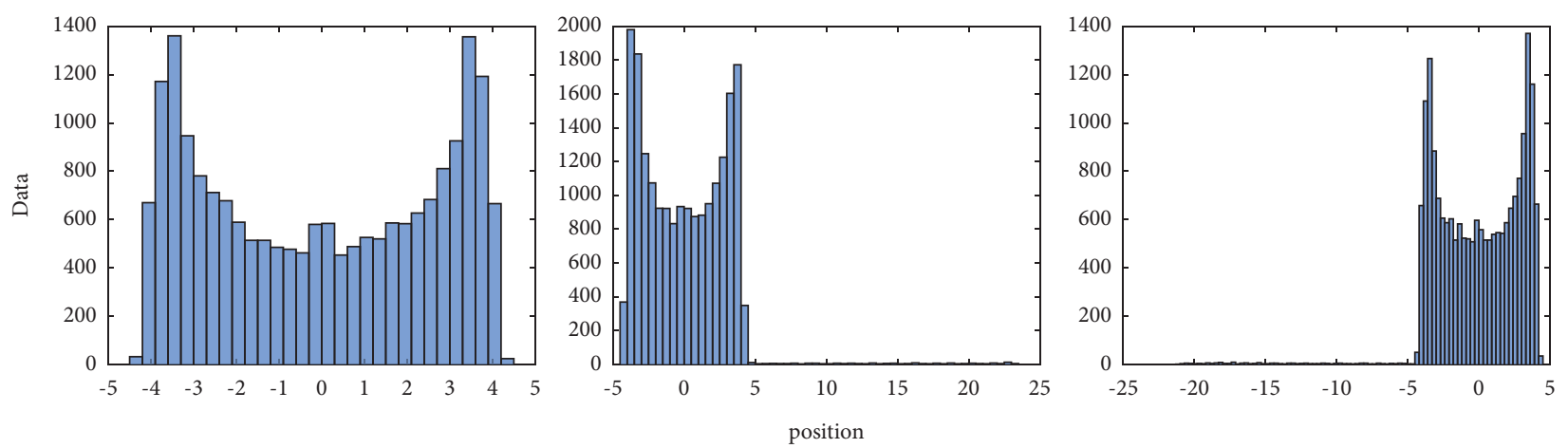

FIgURE 4: Data comparison.
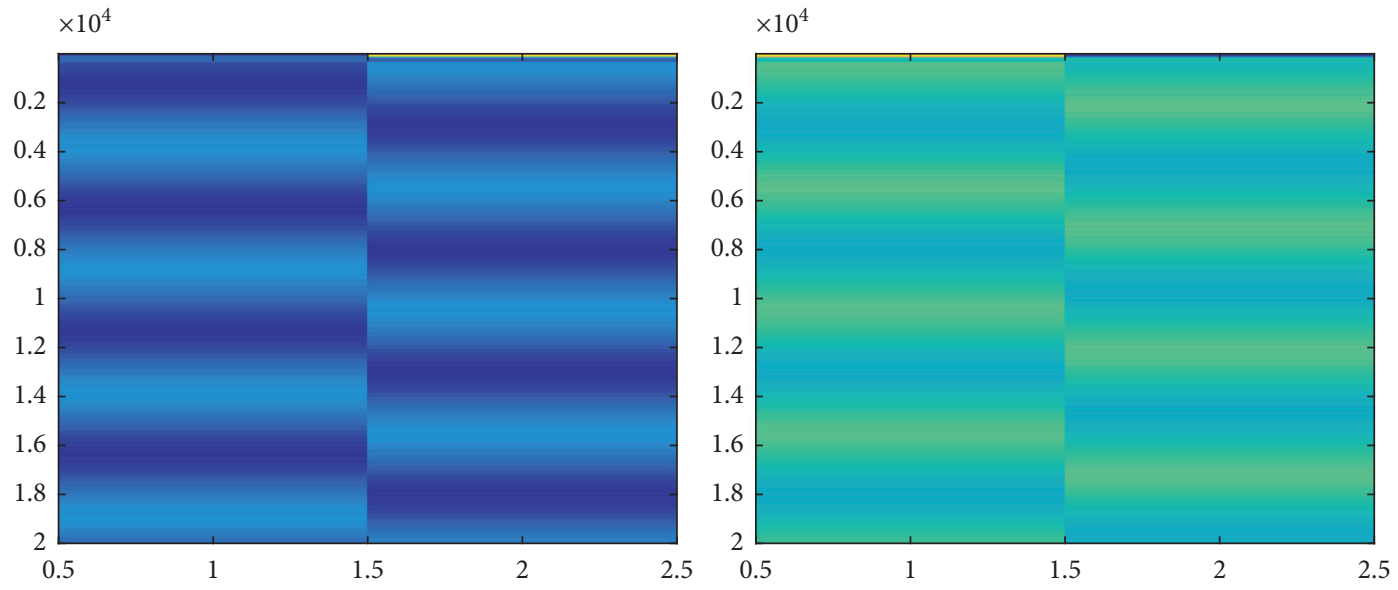

FIgURe 5: Prediction.

(7) Return to step (2) until the adjustment amount of each connection right becomes small. When a model that is very similar to this learning model is provided to the network, it will also encourage the same neuron to win the competition. Therefore, when the network recalls, the input mode can be classified according to equation (9) according to the memorized learning mode; that is, the classification result is represented by the winning neuron of the competition layer. Through the above learning steps, it can be seen that: (1) the learning rate $\eta$ in equation (4) reflects the size of the connection weight adjustment in the learning process, and the typical value of $\eta$ is generally 0.01 to 0.03 . (2) It can be seen from equation (5) that when $P i$ is 1 , the connection weight $w_{i j}$ between the winning neuron $j$ and the input neuron $i$ in the competition layer has $w_{i j}<1$ under the constraint condition of equation (1), so its adjustment is positive; that is, the connection weight changes in the increasing direction; when $P_{i}$ is 0 , the 


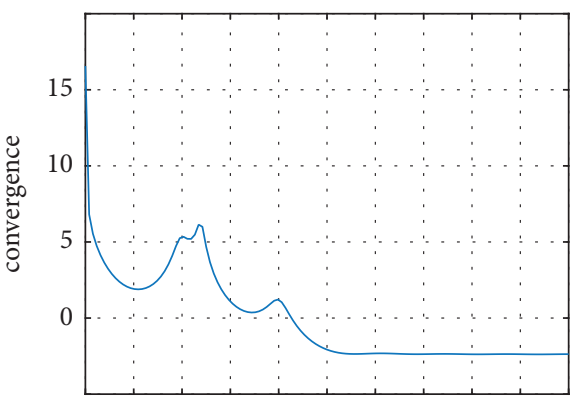

$\begin{array}{lllllllllll}0 & 0.1 & 0.2 & 0.3 & 0.4 & 0.5 & 0.6 & 0.7 & 0.8 & 0.9\end{array}$

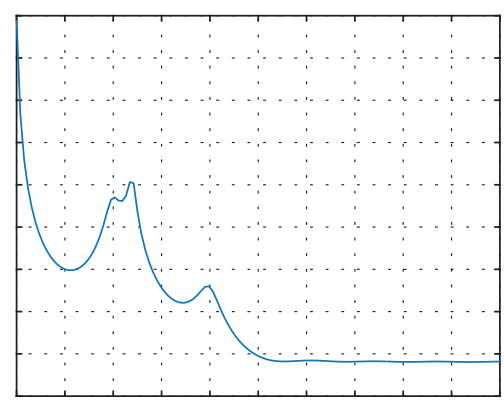

$\begin{array}{lllllllll}0.1 & 0.2 & 0.3 & 0.4 & 0.5 & 0.6 & 0.7 & 0.8 & 0.9\end{array}$

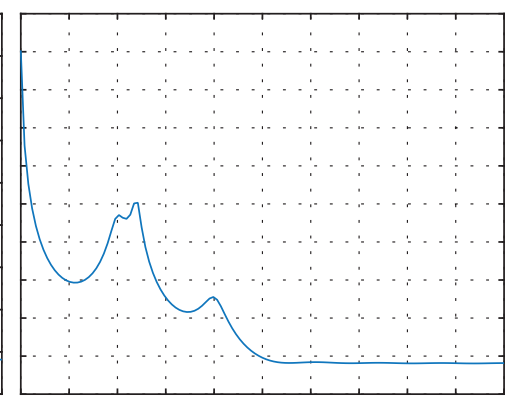

$\begin{array}{lllllllllll}0 & 0.1 & 0.2 & 0.3 & 0.4 & 0.5 & 0.6 & 0.7 & 0.8 & 0.9 & 1\end{array}$

Figure 6: Convergence.

adjustment is negative; that is, the connection weight changes in the decreasing direction. All connection rights always change between $(0,1)$. (3) When the same learning mode is repeatedly provided to the network, the input value $S_{j}$ of the winning neuron in the previous competition layer corresponding to this mode will gradually increase and continue to maintain its status as the winner. The convergence is shown in Figure 6.

\section{Experiment and Analysis}

Here, based on the actual data of China's macroeconomics, a small-scale fuzzy neural network-based early warning system for China's macroeconomics is established. The system structure adopts the above-mentioned 6-layer fuzzy neural network model. The choice of input volume is based on the principle of considering both economic growth and effective demand and is determined as three indicators of price, consumption, and GDP growth rate. And based on the analysis of the indicators, the input variables are fuzzified. In the universe $U$, three types of sets reflecting the level of economic indicators are defined respectively, namely, prices of A large, A medium, and A small; the consumption level of $\mathrm{B}$ large, $\mathrm{B}$ medium, and $\mathrm{B}$ small; and the GDP growth rate of $\mathrm{C}$ large, $\mathrm{C}$ medium, $\mathrm{C}$ small. The membership function uses the Gaussian function (as shown in Figure 2). The output of the system is divided into two parts; one is the output of early warning signals, and the other is the output of early warning comprehensive index. This model is a small system with only 4 fuzzy sets of outputs, namely, overheating $E$ hot, moderate $E$ medium, supercooling $E$ cold, and comprehensive index of the overall economic situation. The output of the early warning signal is the synthesis of the membership degrees of $E$ hot, $E$ neutral, and $E$ cold, respectively. The composite index output of the early warning system is taken from the precise output of the system and reflects the changes in the composite index of economic conditions. The fuzzy reasoning rules of the system are the "interface" between the model and the expert intelligence. In this system, the reasoning rules come from the knowledge and experience of economists (simulation). The evaluated result is shown in Figure 7. From this, the following conclusions can be drawn: the early warning results of the macroeconomic early warning system based on the fuzzy neural network are based on economic theory and basically conform to the objective reality of macroeconomics. The early warning capability of the system has obvious advantages over traditional methods.

The following conclusions can be drawn from the changing trend of each curve:

(1) The comprehensive prosperity index basically correctly reflects the overall situation of the Chinese economy from 1978 to 1998 , the prosperity situation. Around 1988 and 1994 (from 1993 to 1995), China's macroeconomy was already in a state of overheating, exceeding the warning limit; in 1990, the economy fell, exceeding the warning limit and entering a recession zone. Here, we treat the composite index and GDP growth rate as a unified order of magnitude for comparison. These conditions are consistent with China's actual economic conditions at that time.

(2) Compared with the GDP growth rate curve, the composite index curve is similar to the GDP growth rate curve in the general fluctuation cycle, which shows that the composite index is related to the fluctuation of the GDP growth rate, which is in line with economic laws. From the perspective of the waveform, the waveform of the composite index lags behind the GDP growth rate by about half a year in some areas, which is also a manifestation of the characteristics of the composite index. Because the economic status is a comprehensive reflection of economic characteristics, the GDP growth rate only reflects one or several aspects of the economic status. The theory proves that the changes in the GDP growth rate are generally ahead of the changes in the overall state of the economy.

(3) Judging from the waveform diagram, the waveform of the composite index is relatively steep. Therefore, in conjunction with the upper and lower warning limits and the strength of the early warning signal, it has a better function of predicting the overall economic conditions and predicting turning points. For example, in 1996, my country's GDP growth rate was at a high growth rate of $9.6 \%$. Looking at the GDP growth rate alone, no signs of economic "cooling" can be seen, and the opposite conclusion may even 

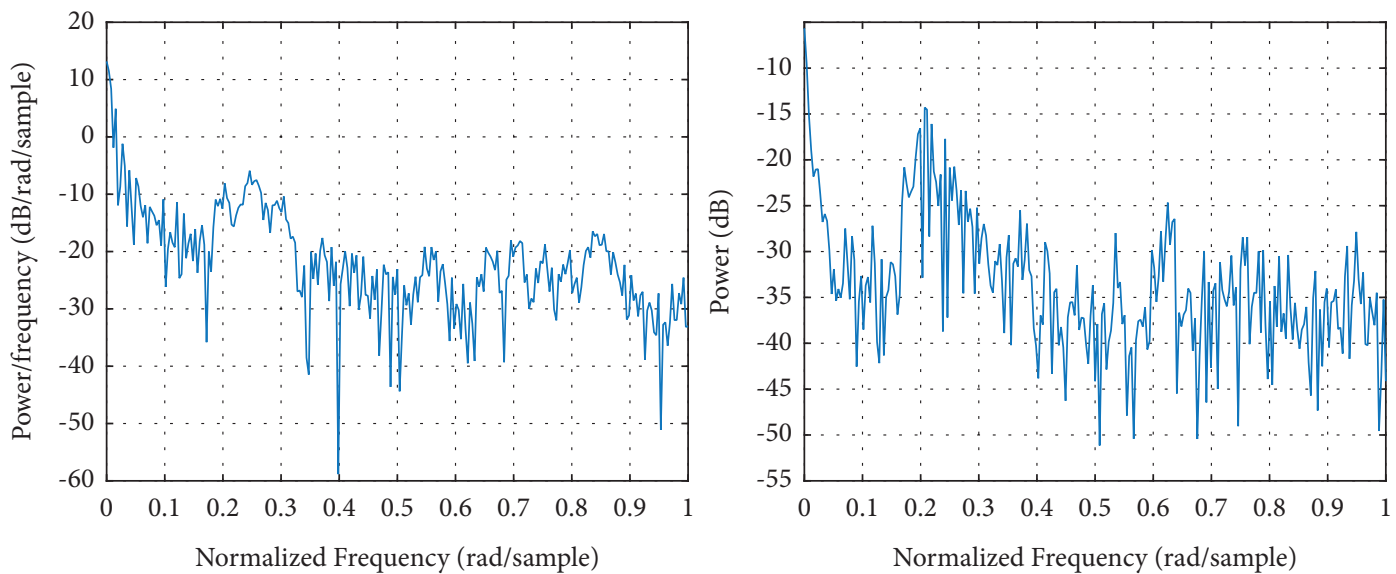

Figure 7: Evaluated result.

be drawn. However, it can be clearly seen from the early warning output that in 1996, my country's economy has clearly turned into a "cold zone."

Using the above-mentioned early warning model to conduct an early warning analysis of the overall economic situation of my country from 1999 to 2001, assuming that there was no excessive external shock during this period, the analysis results are as follows: (1) the overall economic situation of my country has rebounded in 1999. Looking at the relationship between 1998 and 1999, my country's economic situation in 1998 is likely to be the bottom of an economic fluctuation cycle. (2) In 2000, my country's economic situation will continue to improve on the basis of 1999 , but it will not overheat, which is basically a "moderate" situation. (3) In 2001, my country's economic conditions may enter the cold zone again, and in 2000, it may form a peak in the interval from 1998 to 2001.

\section{Conclusion}

This paper proposes to use a self-organizing competitive neural network to select early warning indicators. Its selflearning and adaptive characteristics and fault tolerance overcome the limitations of the above statistical methods:

(1) This paper proposes a method of selecting macroeconomic early warning indicators using self-organizing competitive neural networks and designs a macroeconomic nonlinear early warning model of self-organizing competitive neural networks; fuzzy logic reasoning is used to introduce economic experts' experience into macroeconomic early warning analysis,

(2) The system has the ability to deal with nonlinear and uncertain problems and realizes the intelligence of the early warning process, uses the national macroeconomic indicator data from January 1997 to March 2008 for empirical analysis, and compares the self-organizing competitive neural network method with the traditional KL information method. From the experimental results, compared with the K-L information method, the self-organizing competitive neural network method selects more comprehensive indicators and has greater advantages in seismic resistance and stability.

(3) In addition, this article also demonstrates the effectiveness of this kind of the macroeconomic early warning system through the research and practical simulation application of the macroeconomic early warning system based on the fuzzy neural network.

(4) This type of system realizes the effective combination of objective data and expert experience, increases the amount of information in the early warning system, makes the system reflect a certain "intelligence," and provides new ideas and new methods for the research and application of macroeconomic early warning.

\section{Data Availability}

The data used to support the findings of this study are available from the corresponding author upon request.

\section{Conflicts of Interest}

The authors declare that they have no conflicts of interest.

\section{Acknowledgments}

This work was supported by the Project of Hebei Education Department "Discussion on the transformation and training of accounting professionals under the background of big data, taking the accounting major of Hebei College of Science and Technology as an example" (No. SQ192030)

\section{References}

[1] Y. Yu, C. Yang, Q. Deng, T. Nyima, S. Liang, and C. Zhou, "Memristive network-based genetic algorithm and its application to image edge detection," Journal of Systems Engineering and Electronics, vol. 32, no. 5, pp. 1-9, 2021.

[2] Y. Ishida and S. Hashimoto, "Asymmetric characterization of diversity in symmetric stable marriage problems: an example 
of agent evacuation," Procedia Computer Science, vol. 60, no. 1, pp. 1472-1481, 2015.

[3] P. Zoha and R. Kaushik, "Image edge detection based on swarm intelligence using memristive networks," IEEE Trans. on CAD of Integrated Circuits and Systems, vol. 37, no. 9, pp. 1774-1787, 2018.

[4] J. Pais, "Random matching in the college admissions problem," Economic Theory, vol. 35, no. 1, pp. 99-116, 2018.

[5] J. J. Jung and G. S. Jo, "Brokerage between buyer and seller agents using constraint satisfaction problem models," Decision Support Systems, vol. 28, no. 4, pp. 291-384, 2020.

[6] Y. Liu and K. W. Li, "A two-sided matching decision method for supply and demand of technological knowledge," Journal of Knowledge Management, vol. 21, no. 3, 2017.

[7] J. Byun and S. Jang, "Effective destination advertising: matching effect between advertising language and destination type," Tourism Management, vol. 50, no. 10, pp. 31-40, 2015.

[8] A. N. Nagamani, S. N. Anuktha, N. Nanditha, and V. K. Agrawal, "A genetic algorithm-based heuristic method for test set generation in reversible circuits," IEEE Transactions on Computer-Aided Design of Integrated Circuits and Systems, vol. 37, no. 2, pp. 324-336, 2018.

[9] C. Koch and S. P. Penczynski, "The winner's curse: conditional reasoning and belief formation," Journal of Economic Theory, vol. 174, pp. 57-102, 2018.

[10] C. K. Karl, "Investigating the winner's curse based on decision making in an auction environment," Simulation \& Gaming, vol. 47, no. 3, pp. 324-345, 2016.

[11] D. Ettinger and F. Michelucci, "Creating a winner's curse via jump bids," Review of Economic Design, vol. 20, no. 3, pp. 173-186, 2016.

[12] J. A. Brander and E. J. Egan, “The winner's curse in acquisitions of privately-held firms," The Quarterly Review of Economics and Finance, vol. 65, pp. 249-262, 2017.

[13] Z. Palmowski, “A note on var for the winner's curse," Economics/Ekonomia, vol. 15, no. 3, pp. 124-134, 2017.

[14] B. R. Routledge and S. E. Zin, "Model uncertainty and liquidity," Review of Economic Dynamics, vol. 12, no. 4, pp. 543-566, 2009.

[15] D. Easley and M. O'Hara, "Ambiguity and nonparticipation: the role of regulation," Review of Financial Studies, vol. 22, no. 5, pp. 1817-1843, 2019.

[16] P. Klibano, M. Marinacci, and S. Mukerji, "A smooth model of decision making under ambiguity," Econometrica, vol. 73, no. 6, pp. 1849-1892, 2005.

[17] Y. Halevy, "Ellsberg revisited: an experimental study," Econometrica, vol. 75, no. 2, pp. 503-536, 2017.

[18] D. Ahn, S. Choi, D. Gale, and S. Kariv, "Estimating ambiguity aversion in a portfolio choice experiment," Working paper, vol. 5, no. 2, pp. 195-223, 2019.

[19] T. Hayashi and R. Wada, "Choice with imprecise information: an experimental approach," Theory and Decision, vol. 69, no. 3, pp. 355-373, 2010.

[20] K. Zima, E. Plebankiewicz, and D. Wieczorek, "A SWOT analysis of the use of BIM technology in the polish construction industry," Buildings, vol. 10, no. 1, 2020.

[21] P. Sun, B. Liu, and T. Sun, "Injury status and strategies of female 7-a-side rugby players in Anhui Province," Sports Boutique, vol. 38, no. 3, pp. 72-74, 2019.

[22] P. Guild, M. R. Lininger, and M. Warren, "The association between the single leg hop test and lower-extremity injuries in female athletes: a critically appraised topic," Journal of Sport Rehabilitation, vol. 30, no. 2, pp. 1-7, 2020.
[23] U. G. Inyang, E. E. Akpan, and O. C. Akinyokun, “A hybrid machine learning approach for flood risk assessment and classification," International Journal of Computational Intelligence and Applications, vol. 19, no. 2, Article ID 2050012, 2020.

[24] Q. Liu, S. Du, B. Wyk, and Y. Sun, "Double-layer-clustering differential evolution multimodal optimization by speciation and self-adaptive strategies," Information Sciences, vol. 545, no. 1, pp. 465-486, 2021.

[25] H. R. Medeiros, F. D. Oliveira, H. F. Bassani, and A. Araujo, "Dynamic topology and relevance learning SOM-based algorithm for image clustering tasks," Computer Vision and Image Understanding, vol. 179, pp. 19-30, 2019.

[26] Y. Deng, D. Huang, S. Du, G. Li, and J. Lv, "A double-layer attention based adversarial network for partial transfer learning in machinery fault diagnosis," Computers in Industry, vol. 127, Article ID 103399, 2021.

[27] J. J. Chan, K. K. Chen, S. Sarker et al., "Epidemiology of Achilles tendon injuries in collegiate level athletes in the United States," International Orthopaedics, vol. 44, no. 3, pp. 585-594, 2020.

[28] W. Li, G. G. Wang, and A. H. Gandomi, "A survey of learningbased intelligent optimization algorithms," Archives of Computational Methods in Engineering, vol. 28, pp. 1-19, 2021.

[29] G. G. Wang, A. H. Gandomi, A. H. Alavi, and D. Gong, "A comprehensive review of krill herd algorithm: variants, hybrids and applications," Artificial Intelligence Review, vol. 51, no. 1, pp. 119-148, 2019. 